\title{
Flow dependence of interfacial thermal resistance in nanochannels
}

\author{
Chong Liu, Hai-Bo Fan, Kai Zhang, Matthew M. F. Yuen, and Zhigang Li ${ }^{\text {a) }}$ \\ Department of Mechanical Engineering, The Hong Kong University of Science and Technology, \\ Clear Water Bay, Kowloon, Hong Kong
}

(Received 2 December 2009; accepted 30 January 2010; published online 4 March 2010)

\begin{abstract}
In nanochannel flows, the thermal resistance at the fluid-solid interface may depend on the flow scenario. In this work, we study the interfacial thermal resistance $R_{\mathrm{th}}$ in nanoscale force-driven flows at different temperatures and fluid-wall interactions. For Ar flows in $\mathrm{Cu}$ and $\mathrm{Ag}$ channels, the fluid-wall binding energy is strong and it is found that $R_{\mathrm{th}}$ assumes a maximum value as the external force is varied. The maximum value is caused by the fluid adsorption on the solid surfaces and the temperature increase in the fluid due to viscous frictions. However, when the fluid-wall interaction is weak, the maximum value is not observed and the interfacial thermal resistance decreases monotonously with increasing external force. With the presence of fluid adsorption, it is also found that the peak in $R_{\mathrm{th}}$ is more detectable at low temperature than high temperature. (C) 2010 American Institute of Physics. [doi:10.1063/1.3327931]
\end{abstract}

Thermal management is a key issue in the design of electronic devices. Among the available techniques, the liquid-based cooling methods are quite promising in removing the heat in high-power devices. ${ }^{1-4}$ In certain liquid-based cooling systems, liquid coolants are circulated through micro- or nanoscale channels, which are attached to the heat sources, such as chips. The cooling capacity of such methods depends on the flow rate and thermal resistance at the liquidsolid interface. The flow rate can be controlled by a pump and a high flow rate is usually desired to enhance the cooling capacity. The interfacial thermal resistance $R_{\text {th }}$ depends on many parameters, such as the fluid-wall interaction, surface structure, and temperature. ${ }^{5-10}$ Our recently work indicates that $R_{\mathrm{th}}$ is also associated with the flow rate and the effect of flow rate on the thermal resistance is coupled with the fluidwall interaction and temperature. ${ }^{11}$ However, the detailed information about the dependence of $R_{\mathrm{th}}$ on the flow rate is unavailable. The coupling of the flow rate, fluid-wall interaction, and $R_{\mathrm{th}}$ could be nonlinear and may defy our intuitive understanding that high flow rate improves the cooling capacity. Therefore, it is necessary to explore the relationship between the flow rate and $R_{\mathrm{th}}$ under various conditions. This is also important in the design and optimization of liquidbased cooling techniques.

Compared with experiments, molecular dynamics (MD) simulations offer an easy way to investigate the effects of various parameters on the thermal resistance at the fluidsolid interface. ${ }^{5-15}$ Most of the previous MD studies are mainly focused on how the surface wettability (or fluidsurface binding energy) and structure are related to $R_{\mathrm{th}}$. Little work has been carried out to understand the effect of flow rate on $R_{\mathrm{th}}$. In addition, how the thermal resistance is coupled with the flow rate may be affected by the fluid-surface interaction parameters and the fluid heating due to various

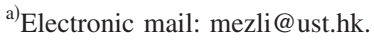

sources of friction. ${ }^{11}$ Although the relationship between the thermal and velocity slips has been numerically investigated, ${ }^{7}$ the dependence of $R_{\mathrm{th}}$ on the flow rate under the effects of surface wettability and fluid heating is not fully understood.

In this study, nonequilibrium MD simulations are conducted to investigate the interfacial thermal resistance in nanoscale force-driven flows. Specifically, liquid Ar flows confined by $\mathrm{Cu}$ and $\mathrm{Ag}$ walls are studied and it is found that $R_{\mathrm{th}}$ assumes a maximum value as the external driving force $F_{\mathrm{e}}$ is varied and the maximum value is more visible at low temperature when the stick boundary condition applies. To understand the effects of fluid-wall interaction on $R_{\mathrm{th}}$, we artificially change the fluid-wall binding energy and found that $R_{\mathrm{th}}$ shows monotonous dependence on the flow rate as the external force is changed when the fluid-wall interaction is weak.

The nanoscale force-driven flow system considered in this work consists of two parallel planar solid walls with a liquid confined in between, as shown in Fig. 1. The wall is modeled by the tight-binding potential function, which has been widely tested and well accepted for transition metals. ${ }^{16,17}$ Particularly, this potential is good for considering the flexibility and energy accommodation of solids. The parameters for $\mathrm{Ag}$ and $\mathrm{Cu}$ are employed in this study for the channel wall. The fluid-fluid interaction is modeled by the Lennard-Jones (LJ) 12-6 potential, $U(r)=4 \varepsilon_{\mathrm{ff}}\left[\left(\sigma_{\mathrm{ff}} / r\right)^{12}\right.$

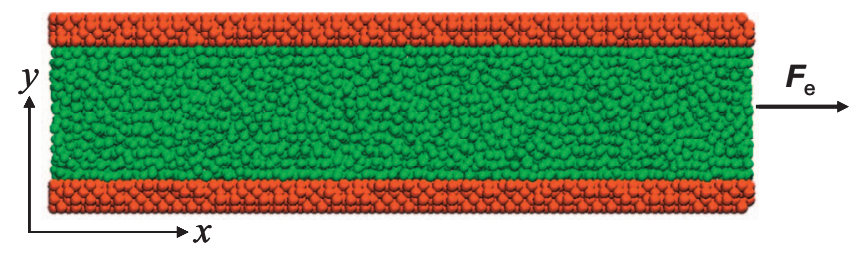

FIG. 1. Structure of a typical force-driven flow system. Red and green particles are wall atoms and fluid molecules, respectively. The external force $F_{\mathrm{e}}$ is applied to each fluid molecule in the positive $x$ direction. 
$\left.-\left(\sigma_{\mathrm{ff}} / r\right)^{6}\right]$, where $r$ is the intermolecular distance, $\varepsilon_{\mathrm{ff}}$ is the fluid-fluid binding energy, and $\sigma_{\mathrm{ff}}$ is the collision diameter of the fluid molecules. The values of $\varepsilon_{\mathrm{ff}}$ and $\sigma_{\mathrm{ff}}$ are set to be $0.0098 \mathrm{eV}$ and $3.47 \AA$, which can be used to describe liquid Ar. ${ }^{18-20}$ The fluid-wall interaction is also calculated by the $\mathrm{LJ}$ potential. To obtain the $\mathrm{Ag}-\mathrm{Ar}$ and $\mathrm{Cu}-\mathrm{Ar}$ interaction parameters in the LJ potential, $\sigma=2.574 \AA$ and $\varepsilon=0.351 \mathrm{eV}$ are adopted for Ag; $\sigma=2.277 \AA$ and $\varepsilon=0.415 \mathrm{eV}$ are used for $\mathrm{Cu}^{21}$ The Lorentz-Berthelot mixing rule is employed to calculate the LJ parameters for the fluid-wall interactions.

The planar walls are perpendicular to the $y$ axis. The initial positions of the wall atoms are obtained by truncating a rectangular portion from a face-centered cubic (fcc) structure with lattice constant equal to 4.086 and $3.615 \AA$ for $\mathrm{Ag}$ and $\mathrm{Cu}$ walls, respectively. Four layers of atoms are used to model the channel wall. The atoms in the outmost layers are fixed to maintain a stable system whereas the atoms in the other three layers are free to vibrate to consider the flexibility of the wall. The channel width (distance between the two innermost layers) $D=4 \mathrm{~nm}$, and the lengths of the system are 19.5 and $5 \mathrm{~nm}$ in the $x$ and $z$ directions, respectively. Fluid molecules are also initially on fcc lattice sites and uniformly distributed in the channel. Random velocities that follow the Maxwell-Boltzmann distribution corresponding to the desired temperature are assigned to the wall atoms and fluid molecules, respectively. Periodic boundary conditions are employed in the $x$ and $z$ directions only. The fluid is moving under an external force, which is applied to each fluid molecule in the $x$ direction. The potential is truncated at $10.21 \AA$ and Newton's equations are integrated with Beeman's algorithm. ${ }^{17,22}$ The time step $\Delta t$ is equal to $1 \mathrm{fs}$. The wall temperature is controlled by scaling the atom velocities. This is important in calculating the heat flow through the wall, as will be discussed later. At the very beginning, the system is relaxed for $100 \mathrm{ps}$ to reach equilibrium. After the equilibration, a force is applied to each fluid molecule in the positive $x$ direction. This external force is linearly increased with time until it reaches the desired value in $50 \mathrm{ps}$. Afterward, the system is allowed to equilibrate for another $100 \mathrm{ps}$ before the temperature distribution and other properties of the fluid are measured. ${ }^{11}$

The temperature of the fluid can be obtained based on the kinetic energy of the fluid molecules. It can also be determined by using the configurational definitions, which do not require the determination of the mean velocity of the fluid. ${ }^{23,24}$ However, the latter is not reliable for calculating the temperature close to the wall due to the fluctuations in fluid density. ${ }^{23}$ For evaluating the interfacial thermal resistance, the fluid temperature near the wall is very important. Therefore, in this work, the temperature of the fluid is measured based on the thermal motion of the fluid. To obtain the temperature distribution, the fluid is divided into $N_{\mathrm{B}}$ bins in the $y$ direction and the temperature in the $i^{\text {th }}$ bin, $T_{i}$, is calculated based on the peculiar velocity of each molecule relative to the streaming velocity of the molecules in the bin,
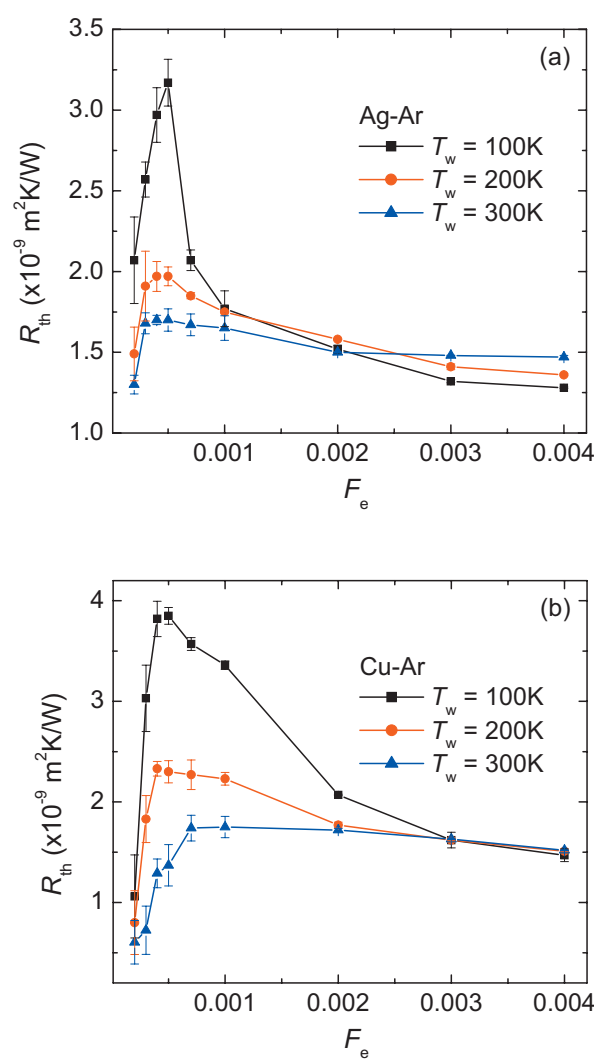

FIG. 2. Interfacial thermal resistance as a function of external driving force at different wall temperatures $T_{\mathrm{w}}$ for Ar flows in $\mathrm{Ag}$ channels (a) and $\mathrm{Cu}$ channels (b). For the external force $F_{\mathrm{e}}, 1$ in MD unit is equal to $1.6 \mathrm{nN}$.

$$
T_{i}=\sum_{j=1}^{N_{i}} m\left(v_{j}-\bar{v}_{i}\right)^{2} /\left(3 N_{i}-\delta\right) k,
$$

where $m$ and $v_{j}$ are the mass and laboratory velocity of each molecule in the bin, $N_{i}$ and $\bar{v}_{i}$ are the total number and mean velocity of the molecules in the bin, $k$ is the Boltzmann constant, and $\delta$ is the number of degrees of freedom used up in determining the mean velocity, which is small compared to $N_{i}$. It is noted that the bin size may affect the temperature distribution. Several values for $N_{\mathrm{B}}$ were used and it was found that $N_{\mathrm{B}}=20$ was appropriate. An alternative method, which is suitable for systems of planar geometry, can also be used to calculate the temperature distribution. ${ }^{25}$

The velocities of all the vibrating wall atoms are scaled at each time step in order to maintain the wall temperature $T_{\mathrm{w}}$ at a desired value. The total kinetic energy, $E$, of the vibrating atoms in the wall and the difference between this total energy and the energy corresponding to the desired wall temperature $T_{\mathrm{w}}, \Delta E=E-3 N k T_{\mathrm{w}} / 2$, are calculated at each step before the velocities of the wall atoms are scaled, where $N$ is the total number of vibrating atoms in the wall. The heat flux through the wall $q$ is calculated based on the average of the energy difference, $q=\langle\Delta E\rangle /(\Delta t \cdot A)$, with $A$ being the area of the wall. The thermal resistance at the fluid-wall interface is estimated by

$$
R_{\mathrm{th}}=\Delta T / q,
$$

where $\Delta T$ is the temperature difference between the wall and the fluid bin near the wall. It is noted that the velocity scaling 

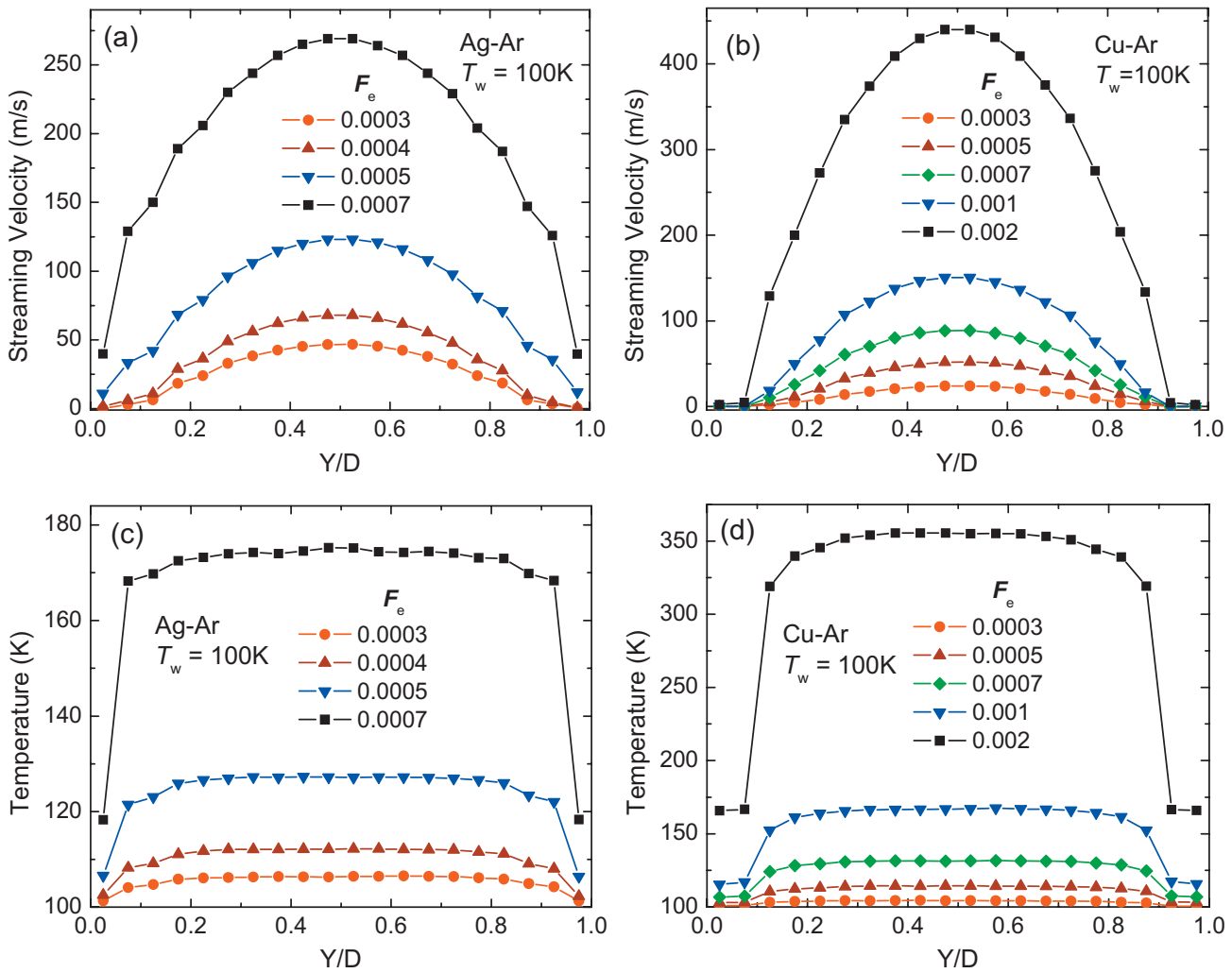

FIG. 3. Velocity and temperature profiles of liquid $\mathrm{Ar}$ in $\mathrm{Ag}$ and $\mathrm{Cu}$ nanochannels under different external driving forces. (a) and (b) are the velocity profiles in $\mathrm{Ag}$ and $\mathrm{Cu}$ channels, respectively. (c) and (d) are the temperature distributions in $\mathrm{Ag}$ and $\mathrm{Cu}$ channels.

may cause the thermal motion of the wall atoms unrealistic. However, our previous work shows that the temperature distribution of the fluid is insensitive to the method of controlling the wall temperature. ${ }^{20}$

The flow rate and velocity are varied by changing the external driving force $F_{\mathrm{e}}$. For the convenience of discussions, the interfacial thermal resistance $R_{\text {th }}$ for Ar flows in $\mathrm{Ag}$ and $\mathrm{Cu}$ channels is plotted as a function of the external force $F_{\mathrm{e}}$ for different wall temperatures, as shown in Fig. 2 (for $F_{\mathrm{e}}$, 1 is equal to $1.602 \mathrm{nN}$ ). It is seen that there is a maximum value in $R_{\mathrm{th}}$ as $F_{\mathrm{e}}$ is varied for both $\mathrm{Ag}$ and $\mathrm{Cu}$ walls. When $F_{\mathrm{e}}$ is small, $R_{\mathrm{th}}$ increases with increasing external force. To understand the increase in $R_{\mathrm{th}}$, it is necessary to study the fluid structure near the wall and how it is affected by the external force. When the external force is small, it cannot break the fluid-wall binding and a layer of fluid molecules are adsorbed by the wall under the strong fluid-wall binding energy. In this case, the stick boundary condition applies, as depicted by the velocity profiles in Figs. 3(a) and 3(b). Due to the strong wall attraction, the potential distribution around the wall shows arrays of potential wells and barriers, as shown in Fig. 4 for $\mathrm{Cu}-\mathrm{Ar}$ interaction. If the external force was absent, the fluid molecules adsorbed by the wall would prefer to stay at the potential wells and vibrate about their equilibrium positions as long as the temperature is not sufficiently high and the kinetic energy of the adsorbed molecules cannot overcome the potential barriers. Therefore, the adsorbed fluid molecules demonstrate solidlike behavior, as confirmed by the structure of the adsorbed fluid layer in Fig. 5 , where the distribution of the fluid molecules is strongly affected by the lattice structure of the wall. With the presence of the external force, the fluid molecules in the adsorbed layer tend to be driven out of the potential wells and the potential energy of the molecules increases. This process is associated with a deduction in the vibrational mobility of the fluid molecules. This is somewhat similar to a spring under tension or compression. As the elastic potential energy of a spring increases, the flexibility of the spring decreases. The $y$-direction trajectories of typical adsorbed Ar molecules confined by $\mathrm{Cu}$ walls, as shown in Fig. 6, confirm that the vibrational mobility of the molecules decreases as the external force is increased. The reduced mobility leads to relatively rigid motion and weakens the momentum and energy transfer

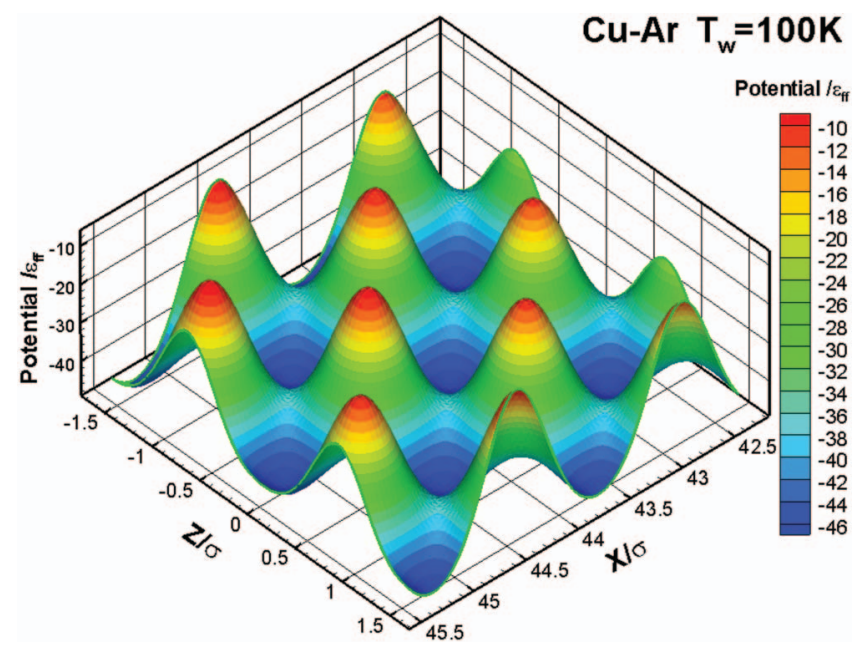

FIG. 4. Three-dimensional contour plot of the potential distribution in the plane near and parallel to $\mathrm{Cu}$ surface. 
(a)

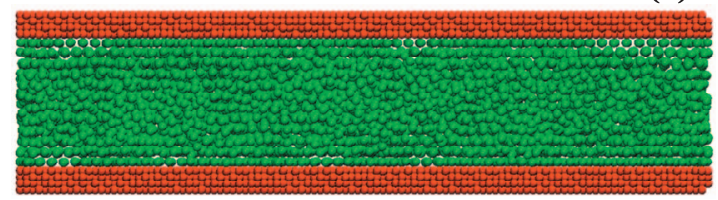

(b)

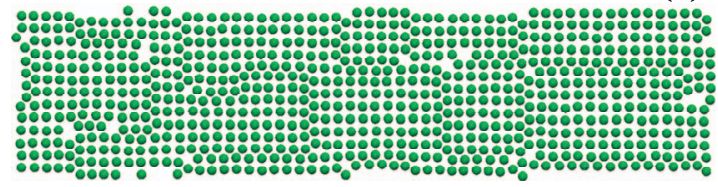

FIG. 5. A snapshot of the fluid structure in a $\mathrm{Cu}$ nanochannel without external force. (a) The side view of the fluid molecules in the nanochannel. (b) The top view of the structure of the adsorbed fluid layer, which is affected by the wall lattices.

between the fluid and wall. This is why $R_{\mathrm{th}}$ increases when the external force is small.

As the external force is increased, after the maximum point, the energy transferred to the flow system in the form of work done by the external force is increased and the viscous friction in the fluid causes fluid heating and raises the temperature of the fluid, ${ }^{11}$ as illustrated by the temperature profiles in Figs. 3(c) and 3(d). ${ }^{26}$ As a consequence, the thermal motion of the fluid molecules is strengthened, which enhances the energy and momentum transfer at the interface and the interfacial thermal resistance decreases.
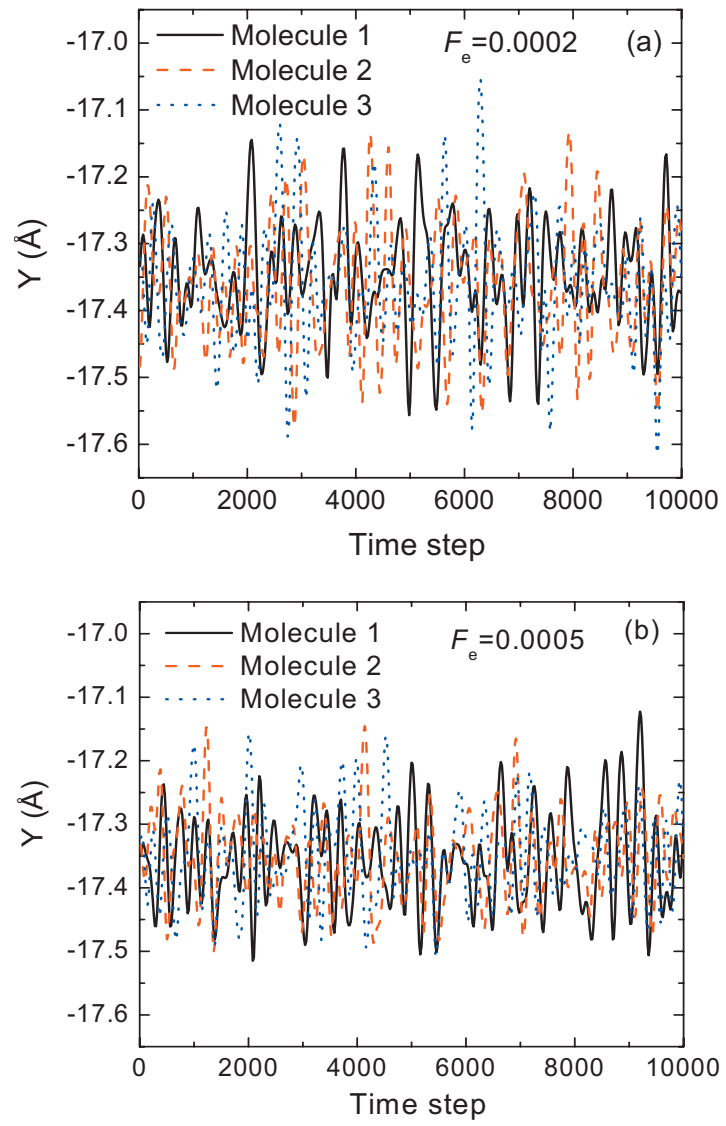

FIG. 6. The $y$-component trajectories of three typical fluid molecules in the absorbed layer under different external forces. (a) $F_{\mathrm{e}}=0.0002$ and (b) $F_{\mathrm{e}}$ $=0.0005$.
When the external force is increased further, it can break the fluid-wall binding and leads to velocity slip at the interface. The velocity slip, on one hand, can result in an increase in $R_{\mathrm{th}}{ }^{7}$ and on the other hand, can greatly raise the fluid temperature through the heat generated by the friction at the fluid-solid interface and reduce $R_{\mathrm{th}}$. When the role of flow slip in reducing $R_{\mathrm{th}}$ is comparable with its contribution to increase $R_{\mathrm{th}}, R_{\mathrm{th}}$ levels off, as shown in Fig. 2, at large external forces. In addition, when there is a clear velocity slip, the fluid temperature becomes much higher than the wall temperature (see Fig. 3) and $R_{\text {th }}$ mainly depends on the external force rather than the wall temperature. This is why $R_{\text {th }}$ becomes independent of wall temperature at large $F_{\mathrm{e}}$.

In Fig. 2, it is also noted that the peak in $R_{\mathrm{th}}$ becomes less detectable as the wall temperature increases. The role of wall temperature lies in the kinetic energy of the fluid molecules near the wall. At high wall temperatures, the kinetic energy of the fluid near the wall is large and thermal motion of the fluid is relatively strong, which help the adsorbed fluid molecules overcome the potential barriers and move freely under relatively small external forces. At low temperature, the thermal motion of the fluid molecules contributes little to the desorption of the fluid molecules, which, in this case, requires a large external force. Hence, $R_{\mathrm{th}}$ increases for a wider range of $F_{\mathrm{e}}$ at low temperature than high temperature.

Furthermore, it is seen in Fig. 2 that $R_{\text {th }}$ has a larger temperature-sensitive range in the external force (from 0 to 0.002) for $\mathrm{Cu}-\mathrm{Ar}$ interface than that for $\mathrm{Ag}-\mathrm{Ar}$ interface (from 0 to 0.0007). This depends on where the velocity slip occurs. As discussed in the previous paragraphs, the wall temperature affects $R_{\mathrm{th}}$ when the stick boundary condition applies, while $R_{\mathrm{th}}$ becomes relatively independent of $T_{\mathrm{w}}$ if there is a slip at the interface. For $\mathrm{Cu}-\mathrm{Ar}$ interaction, the binding energy $\varepsilon_{\mathrm{Cu}-\mathrm{Ar}}=0.064 \mathrm{eV}$, which is larger than the $\mathrm{Ag}-\mathrm{Ar}$ binding energy $\varepsilon_{\mathrm{Ag}-\mathrm{Ar}}=0.058 \mathrm{eV}$. To break the fluidwall binding, the $\mathrm{Cu}-\mathrm{Ar}$ interaction requires a larger external force than the $\mathrm{Ag}-\mathrm{Ar}$ interaction. The stick boundary condition is valid at the $\mathrm{Cu}-\mathrm{Ar}$ interface for $F_{\mathrm{e}}$ up to 0.002 , while the flow slip tends to occur when $F_{\mathrm{e}}$ reaches 0.0005 at $\mathrm{Ag}-\mathrm{Ar}$ interface, as indicated by the velocity profiles in Fig. 3.

We have studied the interfacial thermal resistance for $\mathrm{Ar}$ flows confined by $\mathrm{Cu}$ and $\mathrm{Ag}$ walls. For $\mathrm{Cu}-\mathrm{Ar}$ and $\mathrm{Ag}-\mathrm{Ar}$

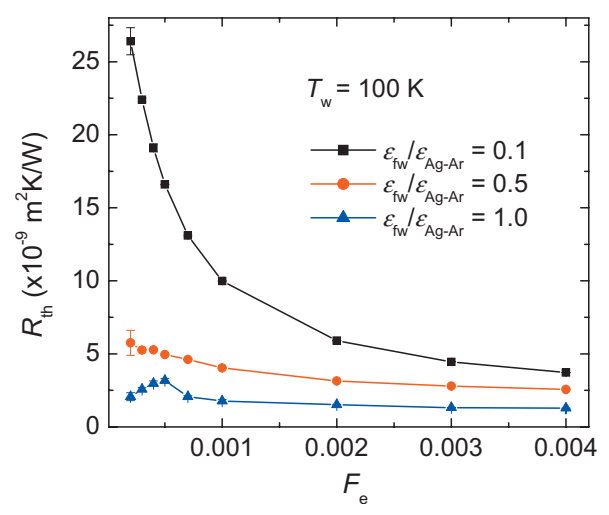

FIG. 7. The dependence of $R_{\mathrm{th}}$ on $F_{\mathrm{e}}$ for different fluid-wall binding energy ratios, $\varepsilon_{\mathrm{fw}} / \varepsilon_{\mathrm{Ag}-\mathrm{Ar}}$, in the $\mathrm{Ag}-\mathrm{Ar}$ flow system at $T_{\mathrm{w}}=100 \mathrm{~K}$. 


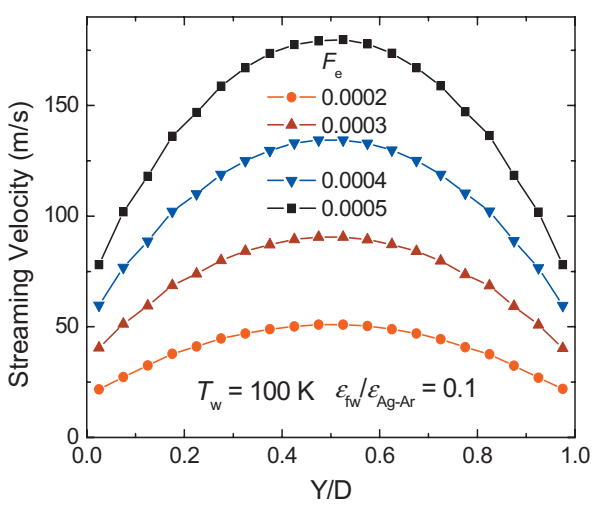

FIG. 8. Velocity distribution of Ar at different external driving forces for $\varepsilon_{\mathrm{fw}} / \varepsilon_{\mathrm{Ag}-\mathrm{Ar}}=0.1$ and $T_{\mathrm{w}}=100 \mathrm{~K}$ in the $\mathrm{Ag}-\mathrm{Ar}$ flow system.

interactions, the binding energy is quite large and the fluid adsorption is a popular phenomenon at the interface at or below room temperature. However, if the fluid-wall binding is weak, the fluid adsorption may not be favored at the interface. In this situation, the dependence of $R_{\mathrm{th}}$ on the flow could be different from the cases shown in Fig. 2. To understand the variation in $R_{\text {th }}$ under weak fluid-wall interactions, we artificially change the fluid-wall binding energy $\varepsilon_{\mathrm{fw}}$ in the $\mathrm{Ag}-\mathrm{Ar}$ system at $T_{\mathrm{w}}=100 \mathrm{~K}$. Figure 7 shows $R_{\mathrm{th}}$ as a function of $F_{\mathrm{e}}$ at different $\varepsilon_{\mathrm{fw}} / \varepsilon_{\mathrm{Ag}-\mathrm{Ar}}$ values. It is found that the maximum point disappears when $\varepsilon_{\mathrm{fw}}$ is small and $R_{\mathrm{th}}$ monotonously decreases as the external force is increased. For weak fluid-wall interactions, fluid adsorption is not observed even if the external force is absent and there is always a velocity slip when an external force is applied, as depicted by the velocity profiles in Fig. 8. Therefore, the increasing thermal resistance shown in Fig. 2 due to fluid adsorption is not detected. The mechanism of decreasing $R_{\mathrm{th}}$ with increasing external force for small fluid-wall interactions is the same as that in Fig. 2 after the maxima.

In summary, we have investigated the variation of interfacial thermal resistance in nanochannel flows as the external driving force is changed. For Ar flows confined by $\mathrm{Cu}$ and $\mathrm{Ag}$ walls, the fluid-wall interaction is strong and it is found that $R_{\mathrm{th}}$ assumes a maximum value as the external driving force is increased. For weak fluid-wall binding energies, the fluid adsorption is not observed and $R_{\mathrm{th}}$ decreases monotonously with increasing external force.

This work was supported by the Hong Kong Innovation and Technology Fund under Grant No. GHP/035/07GD.

${ }^{1}$ S. V. Garimella and C. B. Sobhan, Annu. Rev. Heat Transfer 13, 1 (2003).

${ }^{2}$ R. B. Oueslati, D. Therriault, and S. Martel, IEEE Trans. Compon. Packag. Technol. 31, 869 (2008).

${ }^{3}$ E. Baird and K. Mohseni, IEEE Trans. Compon. Packag. Technol. 31, 143 (2008).

${ }^{4}$ L. L. Vasiliev, Appl. Therm. Eng. 25, 1 (2005).

${ }^{5}$ B. H. Kim, A. Beskok, and T. Cagin, J. Chem. Phys. 129, 174701 (2008).

${ }^{6}$ A. J. Markvoort, P. A. J. Hilbers, and S. V. Nedea, Phys. Rev. E 71, 066702 (2005).

${ }^{7}$ R. Khare, P. Keblinski, and R. Yethiraj, Int. J. Heat Mass Transfer 49, 3401 (2006).

${ }^{8}$ M. Shibahara, T. Kunugi, K. Muko, and M. Katsuki, Nanoscale Microscale Thermophys. Eng. 10, 197 (2006).

${ }^{9}$ M. Shibahara and K. Takeuchi, Nanoscale Microscale Thermophys. Eng. 12, 311 (2008).

${ }^{10}$ G. Balasubramanian, S. Banerjee, and I. K. Puri, J. Appl. Phys. 104, 064306 (2008).

${ }^{11}$ Z. Li, Phys. Rev. E 79, 026312 (2009).

${ }^{12}$ S. Maruyama and T. Kimura, Therm. Sci. Eng. 7, 63 (1999).

${ }^{13}$ T. Ohara and D. Suzuki, Nanoscale Microscale Thermophys. Eng. 4, 189 (2000).

${ }^{14}$ L. Xue, P. Keblinski, and S. R. Phillpot, S. U. Choi, and J. A. Eastman, J. Chem. Phys. 118, 337 (2003).

${ }^{15}$ C. S. Wang, J. S. Chen, J. Shiomi, and S. Maruyama, Int. J. Therm. Sci. 46, 1203 (2007).

${ }^{16}$ F. Cleri and V. Rosato, Phys. Rev. B 48, 22 (1993).

${ }^{17}$ Z. Li and H. Wang, Phys. Rev. Lett. 95, 014502 (2005).

${ }^{18}$ H. Hippler, J. Troe, and H. J. Wendelken, J. Chem. Phys. 78, 6709 (1983).

${ }^{19}$ C. Liu and Z. Li, Phys. Rev. E 80, 036302 (2009).

${ }^{20}$ C. Liu and Z. Li, J. Chem. Phys. 132, 024507 (2010).

${ }^{21}$ P. M. Agrawal, B. M. Rice, and D. L. Thompson, Surf. Sci. 515, 21 (2002).

${ }^{22}$ Z. Li, Phys. Rev. E 80, 061204 (2009).

${ }^{23}$ J. Delhommelle and D. J. Evans, J. Chem. Phys. 114, 6229 (2001).

${ }^{24}$ A. Baranyai, J. Chem. Phys. 112, 3964 (2000).

${ }^{25}$ P. J. Daivis, K. P. Travis, and B. D. Todd, J. Chem. Phys. 104, 9651 (1996).

${ }^{26}$ It is noted that the velocity and temperature profiles in nanochannel flows generally deviate from the predictions of the classical Navier-Stokes equations due to the boundary conditions and inhomogeneous fluid properties. Further information can be found in Ref. 11 for temperature distribution and Ref. 19 for velocity distribution. 